\title{
Atrial Arrhythmia Ablation in Adult Congenital Heart Disease with a Persistent Left-side Superior Vena Cava
}

\author{
Shinya Yamada ${ }^{1,2}$, Li-Wei Lo ${ }^{1,3}$, Yenn-Jiang Lin ${ }^{1,3}$, Atul Prabhu ${ }^{1}$ and Shih-Ann Chen ${ }^{1,3}$
}

\begin{abstract}
:
We herein report a case of atrial tachycardia (AT) originating from the dilated coronary sinus (CS) connected to a persistent left superior vena cava. The earliest activation site of AT was localized at the superior CS ostium, identified using a novel ultra-rapid high-density mapping system (Rhythmia ${ }^{\mathrm{TM}}$ ). Successful ablation was performed at the corresponding position. This report demonstrated the utility of an ultra-rapid highdensity mapping system in identifying arrhythmogenic foci in adult cases of congenital heart disease.
\end{abstract}

Key words: ultra-rapid high-density mapping, atrial tachycardia, adult congenital heart disease, dilated coronary sinus

(Intern Med 56: 3051-3055, 2017)

(DOI: 10.2169/internalmedicine.8492-16)

\section{Introduction}

The coronary sinus (CS) ostium is a recognized site of atrial tachycardia (AT) origin (1). Rarely, CS anomalies, such as connection to the left superior vena cava (SVC) and enlargement of the CS ostium, contribute to the development of atrial arrhythmogenesis (2). Radiofrequency (RF) catheter ablation is considered a safe and effective therapeutic strategy for AT (1). However, detailed mapping is necessary to identify the arrhythmogenic substrates in congenital heart disease and cardiac anomalies. Recently, an automatic, ultrarapid high-density mapping system (Rhythmia ${ }^{\mathrm{TM}}$, Boston Scientific, Cambridge, USA) was reported to accurately and rapidly identify geometry and complex patterns of activation in atrial arrhythmias (3). This mapping system may facilitate catheter ablation for complex atrial arrhythmias in adult cases of congenital heart disease.

We herein report a case of AT identified within the giant CS connected to a persistent left SVC using ultra-rapid high-density mapping.

\section{Case Report}

A 32-year-old woman was diagnosed with secundum atrial septal defect post patch closure and persistent left SVC. She had been clinically stable without medical therapy until several recent attacks of severe palpitations, and she visited the emergency department due to a major attack. Fig. 1 shows the 12-lead electrocardiogram before (Fig. 1A) and after (Fig. 1B) adenosine (12 mg) administration. Fig. 1A shows regular and narrow QRS complex tachycardia with a ventricular rate of $210 \mathrm{bpm}$. The tachycardia was not terminated by adenosine administration, but discrete $\mathrm{P}$ waves were found, as shown in Fig. 1B. The P-wave morphology was positive in leads I, II and aVL and negative in lead aVR. These PP intervals were irregular, and the $\mathrm{P}$ wave duration was short. Therefore, she was referred to our department for further treatment of this frequent tachycardia.

Supraventricular tachycardia (SVT) was reproducibly induced or terminated by overdrive atrial pacing during the electrophysiologic study. The earliest atrial activation was observed not in the RA but within the CS. Therefore, ultrarapid high-density mapping was used to identify the earliest activation site of SVT while simultaneously reconstructing

\footnotetext{
${ }^{1}$ Division of Cardiology, Department of Medicine, Taipei Veterans General Hospital, Taiwan, ${ }^{2}$ Department of Cardiovascular Medicine, Fukushima Medical University, Japan and ${ }^{3}$ Institute of Clinical Medicine, and Cardiovascular Research Institute, National Yang-Ming University, Taiwan

Received: November 2, 2016; Accepted: February 22, 2017; Advance Publication by J-STAGE: September 15, 2017

Correspondence to Dr. Shih-Ann Chen, epsachen@ms41.hinet.net
} 
A)

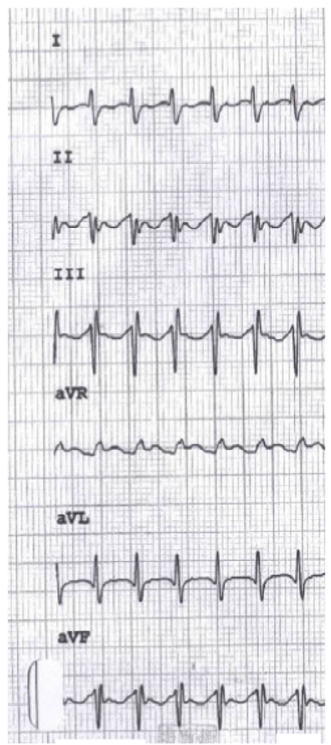

B)

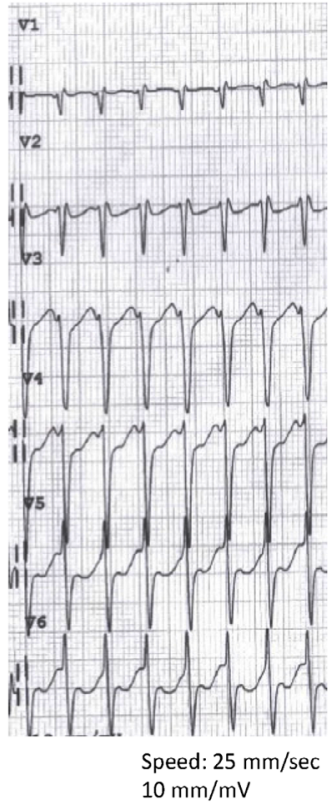

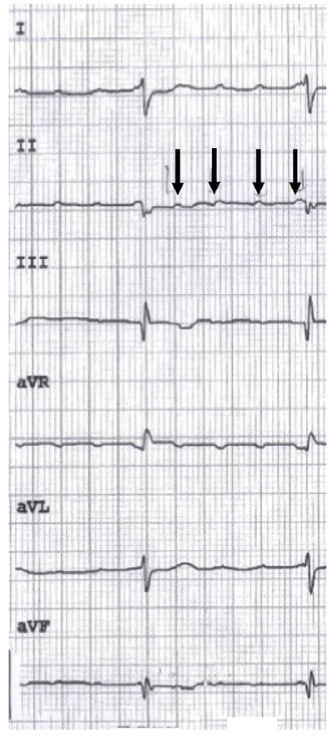

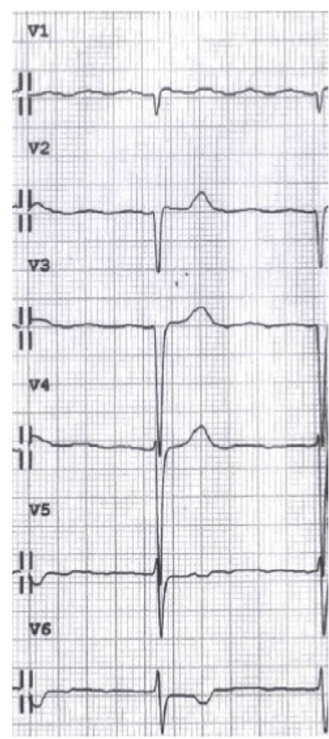

Speed: $25 \mathrm{~mm} / \mathrm{sec}$ $10 \mathrm{~mm} / \mathrm{mV}$

Figure 1. Standard 12-lead electrocardiogram recorded in the emergency department. A: Standard 12-lead electrocardiogram before adenosine administration. Narrow QRS complex tachycardia with a ventricular rate of $210 \mathrm{bpm}$ is noted. B: Standard 12-lead electrocardiogram after adenosine administration (12 mg). The tachycardia is not terminated by adenosine administration, but discrete $P$ waves (arrows) are found.

the CS geometry. In order to collect the mapping data, we used a mini basket catheter (Orion ${ }^{\mathrm{TM}}$, Boston Scientific), which contains 8 splines with 8 electrodes (2.5-mm spacing) per spline. The earliest activation originated from the region of the superior CS ostium and spread centrifugally, suggesting the AT focal pattern shown in Fig. 2A. The earliest activation site of AT, the low-voltage area during sinus rhythm (Fig. 2B), and the QS pattern in unipolar recording during AT (Fig. 2C) were located at the superior CS ostium (mapping time, 2.3 minutes; total mapping points, 4,073 points). In addition, we performed entrainment mapping during AT. Entrainment attempted at the earliest activation site demonstrated concealed entrainment (Fig. 3). Therefore, RF energy was delivered to the corresponding area. AT was not induced by programmed electrical stimulation with or without isoproterenol infusion after multiple shots. CS venography showed a dilated CS, and the CS ostium diameter was 28 mm (Fig. 4).

\section{Discussion}

The CS ostium accounts for approximately $7 \%$ of AT foci (1). This site has complex anatomical structures, which may contribute to the genesis of AT (1). Weiss et al. showed that anomalies of the CS, such as diverticula, persistent left SVC or enlargement of the CS ostium, were found in $9 \%$ of patients with SVT (2). They also showed that the stretch induced by a large CS ostium may modify the electrophysi- ological characteristics of the atrial cardiac tissue. In the present case, the earliest activation site of AT was consistent with the low-voltage-zone border. Although a majority of histological studies conducted on myocardium from AT foci have demonstrated normal voltage findings, abnormal myocardium with endocardial fibrosis and myofiber hypertrophy have also been seen $(4,5)$. A previous study using a threedimensional mapping system showed that focal AT can also originate within or around a low-voltage zone or border zone (6). Therefore, the low-voltage zone around the superior CS ostium may be related to the anatomic changes induced by a large CS ostium and may be a potential source for the production of arrhythmogenic substrates.

A previous report showed that the P-wave morphology during AT was deeply negative in inferior leads and positive in lead aVL at the CS ostium (1). In our case, the P-wave morphology was positive in leads I, II and aVL and negative in lead aVR. Although the P-wave morphology during tachycardia is useful for differentiating the atrium of origin before RF ablation, such differentiation is difficult in patients with cardiac anomalies. Indeed, in the present case, the AT origin around the superior CS ostium was located at the high atrium in contrast to the normal heart (Fig. 2A). Qian et al. reported that a positive P-wave in inferior leads and a negative $\mathrm{P}$ wave in lead aVR indicated a high atrial origin (7).

In their analysis of the AT mechanism, Markowitz et al. reported that microreentrant AT could not be terminated 

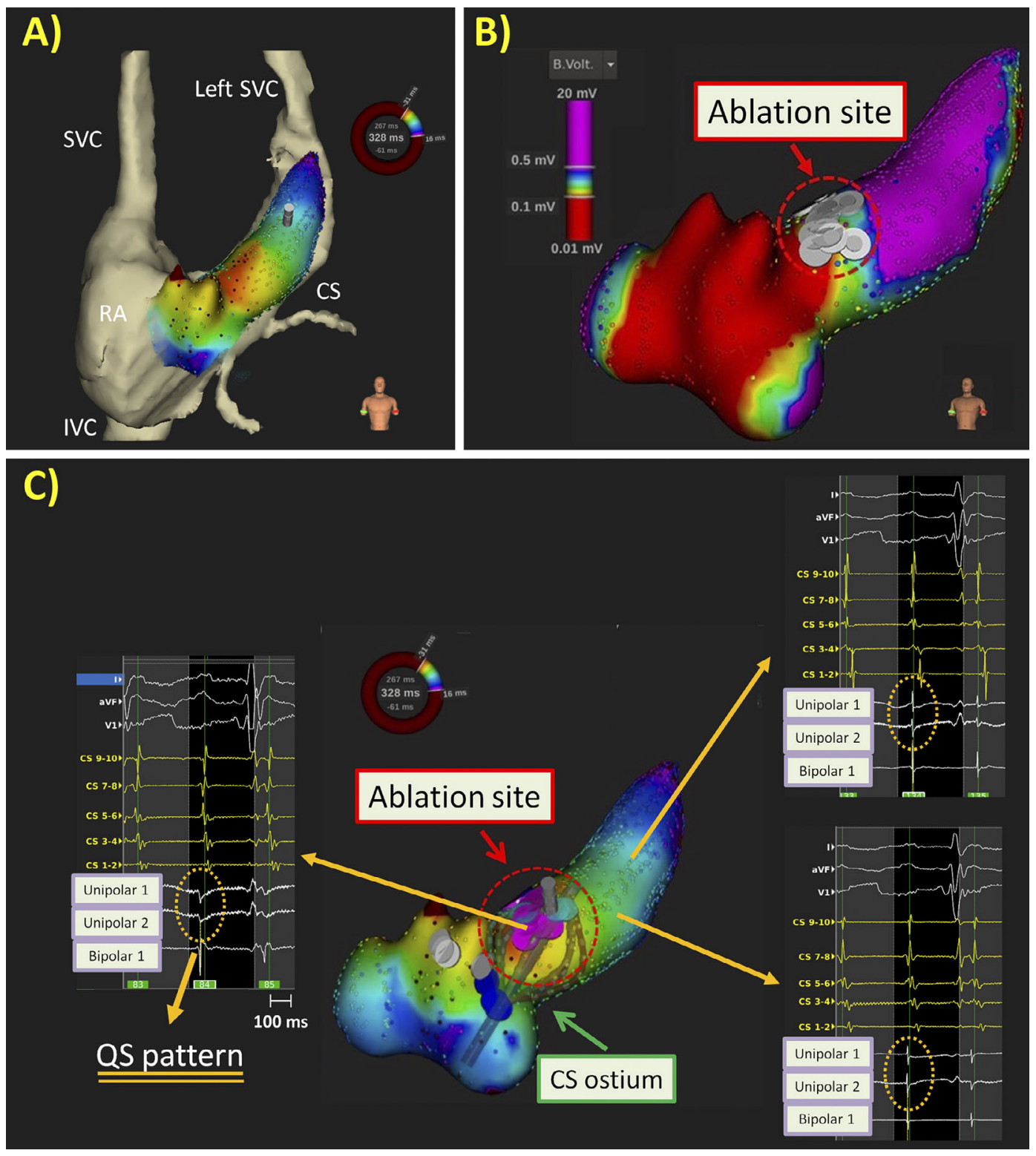

Figure 2. Using ultra-rapid high-density mapping during mapping and ablation procedures. A: The activation time during AT is color-coded within the CS. CS images are merged with computed tomography images. The earliest activation (red) originated from the region of the superior CS ostium and spread centrifugally. Computed tomography shows the dilated CS connected to the persistent left SVC. B: Voltage map during sinus rhythm. Low voltage is defined as $\leq \mathbf{0 . 5} \mathbf{m V}$. Electrogram amplitude $(\mathrm{mV})$ is color-coded from red $(<0.1 \mathrm{mV})$ to yellow, green and blue to purple $(>0.5 \mathrm{mV})$. The successful ablation site is located within the low-voltage zone. C: Local potentials within CS during AT are demonstrated. The QS pattern in a unipolar recording during tachycardia is located at the superior CS ostium. CS: coronary sinus, SVC: superior vena cava, IVC: inferior vena cava, RA: right atrium, AT: atrial tachycardia

with adenosine, and that this tachycardia was induced and/or terminated by programmed electrical stimulation (8). In our case, AT could not be clinically terminated with adenosine. In an electrophysiological study, tachycardia was easily induced and terminated by programmed electrical stimulation. In addition, entrainment attempted from the earliest activation site showed concealed entrainment. The low-voltage zone around the superior CS ostium may be a potential source for the development of microreentrant AT.
Current 3D electroanatomical mapping systems can accurately identify the site of earliest activation in focal tachycardias. However, they require careful point-by-point mapping data acquisition. Ultra-rapid high-density mapping using a mini basket catheter can obtain a high-resolution electroanatomical activation and substrate voltage map rapidly and identify the origin of tachycardia accurately (3). In our case, the mapping time was only 2.3 minutes for a total of 4,073 mapping points. Importantly, this ultra-rapid high- 


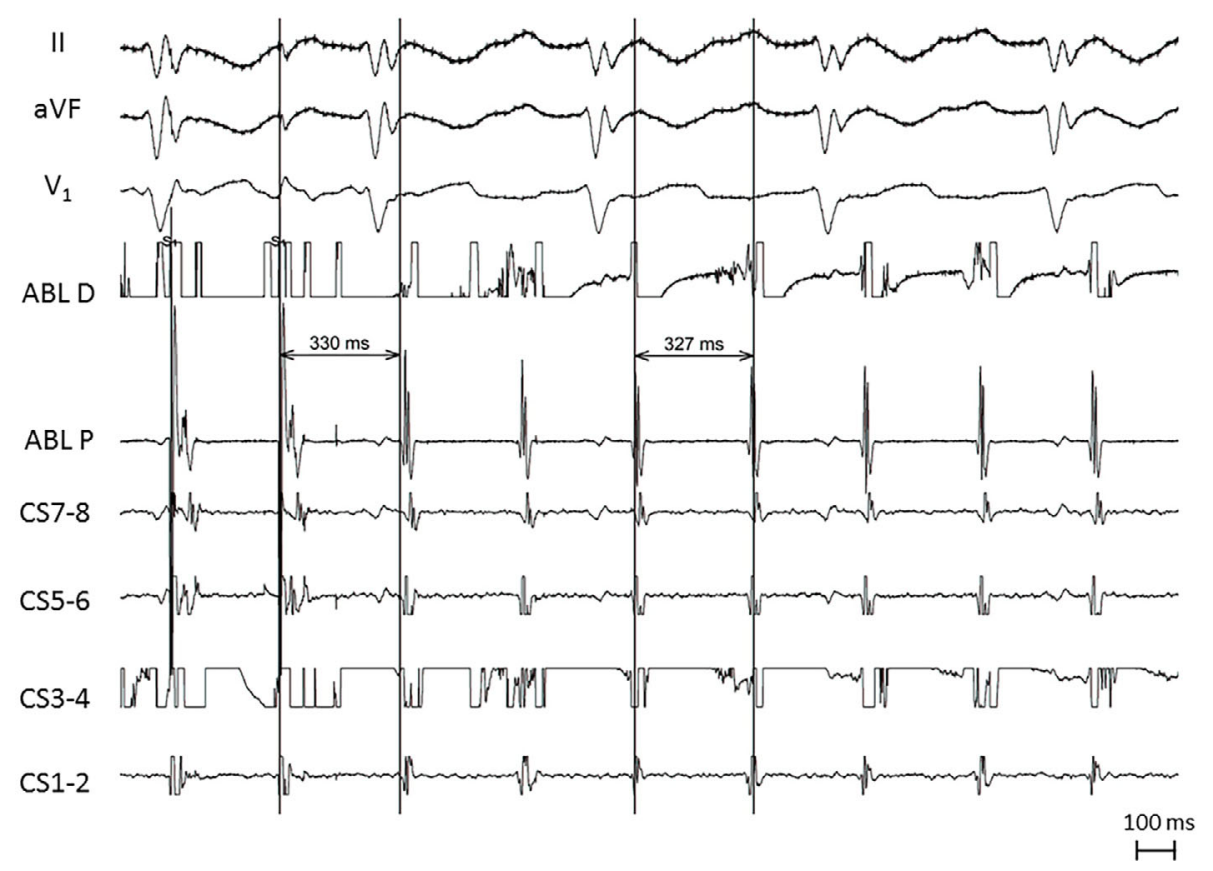

Figure 3. Intracardiac electrogram during entrainment attempted at the earliest activation site. Pacing was performed from the ablation catheter at $\mathbf{3 0 0} \mathrm{ms}$ during atrial tachycardia. The post-pacing interval $(330 \mathrm{~ms})$ was consistent with the tachycardia cycle length $(327 \mathrm{~ms})$. The ablation catheter was located at the earliest activation site during atrial tachycardia. Ablation D: ablation distal, Ablation P: ablation proximal

A)
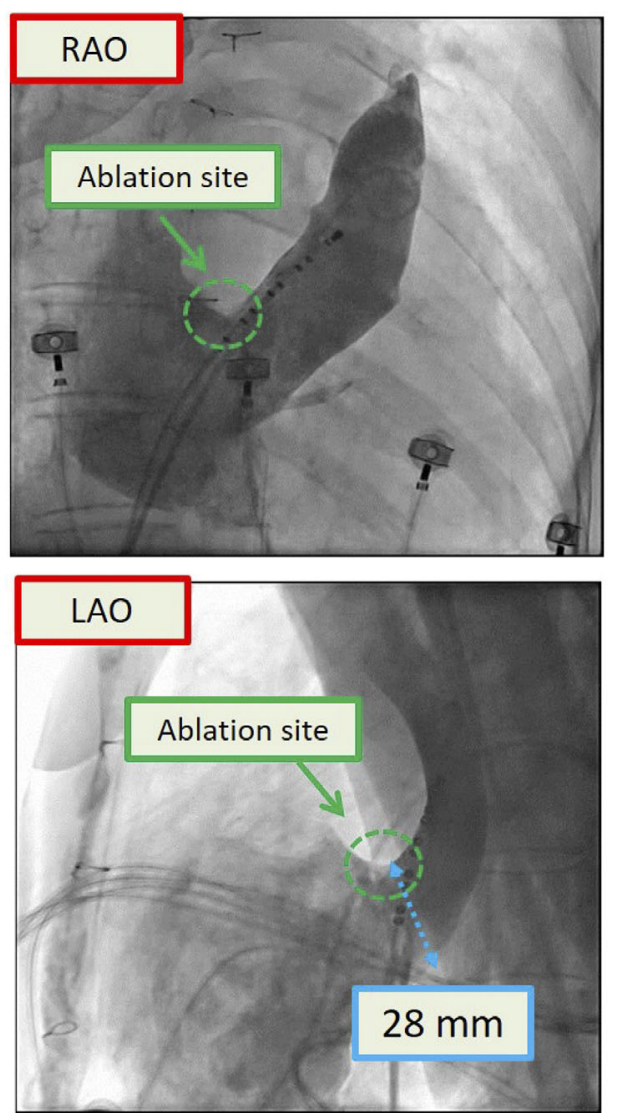

B)
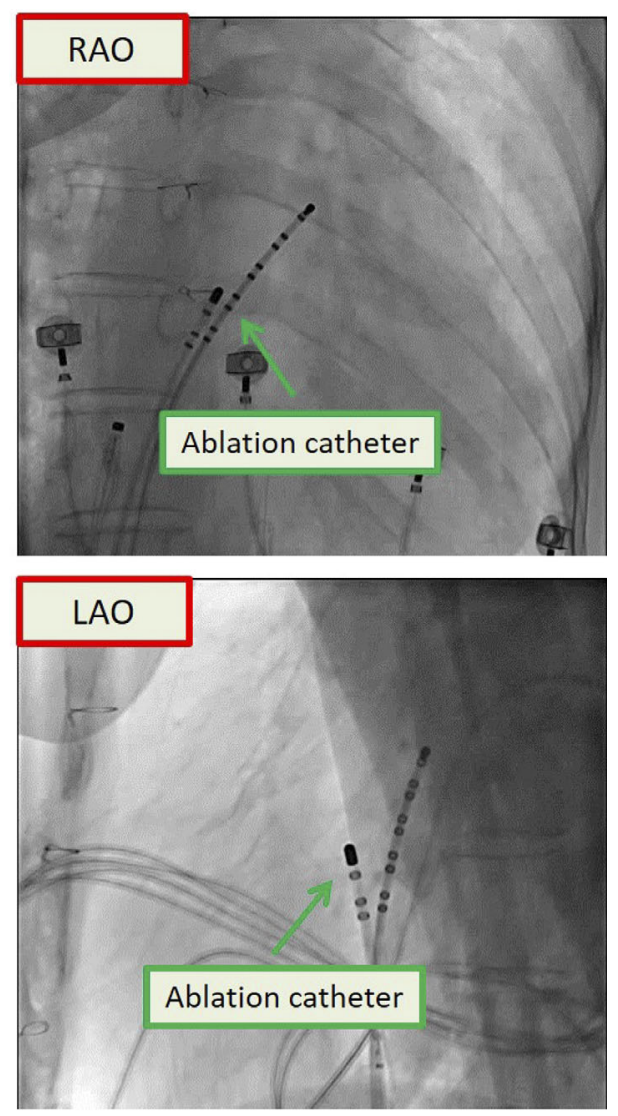

Figure 4. CS venography. A: A multielectrode catheter is placed at the CS. Right and left anterior oblique images reveal the dilated CS. The CS ostium diameter was $28 \mathrm{~mm}$. B: The location of successful radiofrequency catheter ablation. Right and left anterior oblique images show that the ablation catheter was located at the superior CS ostium. CS: coronary sinus 
density mapping system not only identifies the arrhythomgenic substrates for AT within the giant CS connected to persistent left SVC but also provides the local unipolar and bipolar electrogram morphologies.

In conclusion, this mapping system proved useful for mapping and ablation in an adult case of congenital heart and cardiac anomaly.

The authors state that they have no Conflict of Interest (COI).

\section{Acknowledgement}

The present work was supported by the Taipei Veterans General Hospital (V103C-042, V103C-126, V103E7-002, VGHUST 103-G1-3-1, V104C-131, V104E7-003, V105C-060), Ministry of Science and Technology (NSC 102-2325-B-010-005, MOST 1032314-B-075-062-MY3, MOST 104-2314-B-075-065-MY2), and Research Foundation of Cardiovascular Medicine (RFCM 10101-001, 104-01-009-1).

\section{References}

1. Kistler PM, Fynn SP, Haqqani H, et al. Focal atrial tachycardia from the ostium of the coronary sinus: electrocardiographic and electrophysiological characterization and radiofrequency ablation. J Am Coll Cardiol 45: 1488-1493, 2005.
2. Weiss C, Cappato R, Willems S, Meinertz T, Kuck KH. Prospective evaluation of the coronary sinus anatomy in patients undergoing electrophysiologic study. Clin Cardiol 22: 537-543, 1999.

3. Nakagawa H, Ikeda A, Sharma T, Lazzara R, Jackman WM. Rapid high resolution electroanatomical mapping evaluation of a new system in a canine atrial linear lesion model. Circ Arrhythm Electrophysiol 5: 417-424, 2012.

4. McGuire MA, Johnson DC, Nunn GR, Yung T, Uther JB, Ross DL. Surgical therapy for atrial tachycardia in adults. J Am Coll Cardiol 14: 1777-1782, 1989.

5. Rosso R, Kistler PM. Focal atrial tachycardia. Heart 96: 181-185, 2010.

6. Higa S, Tai CT, Lin YJ, et al. Focal atrial tachycardia new insight from noncontact mapping and catheter ablation. Circulation 109: 84-91, 2004.

7. Qian ZY, Hou XF, Xu DJ, et al. An algorithm to predict the site of origin of focal atrial tachycardia. Pacing Clin Electrophysiol 34: 414-421, 2011.

8. Markowitz SM, Nemirovksy D, Stein KM, et al. Adenosineinsensitive focal atrial tachycardia: evidence for de novo micro-reentry in the human atrium. J Am Coll Cardiol 49: 1324-1333, 2007.

The Internal Medicine is an Open Access article distributed under the Creative Commons Attribution-NonCommercial-NoDerivatives 4.0 International License. To view the details of this license, please visit (https://creativecommons.org/licenses/ by-nc-nd/4.0/).

(C) 2017 The Japanese Society of Internal Medicine Intern Med 56: 3051-3055, 2017 